\title{
An accretion based data mining algorithm for identification of sets of correlated neurons \author{
and Sonja Grün ${ }^{2,4}$
} \\ Denise Berger*1,2, Christian Borgelt ${ }^{3}$, Markus Diesmann ${ }^{4}$, George Gerstein ${ }^{5}$
}

\begin{abstract}
Address: ${ }^{1}$ Neuroinformatics, Inst. Biology, Freie Universität, Berlin, 14195, Germany, ${ }^{2}$ Bernstein Center for Computational Neuroscience, Berlin, Germany, ${ }^{3}$ European Center for Soft Computing, Mieres (Asturias), 33600, Spain, ${ }^{4}$ Department of Neuroscience, University of Pennsilvania, Phildelphia, 19104, USA and ${ }^{5}$ Neuroscience Group, RIKEN Brain Science Institute, 2-1 Hirosawa, Wako-shi, 351-0198 Saitama, Japan

Email: Denise Berger* - d_berger@gmx.net

* Corresponding author
\end{abstract}

from Eighteenth Annual Computational Neuroscience Meeting: CNS*2009

Berlin, Germany. 18-23 July 2009

Published: 13 July 2009

BMC Neuroscience 2009, I0(Suppl I):P254 doi:I0.II86/I47I-2202-I0-SI-P254

This abstract is available from: http://www.biomedcentral.com//47I-2202//0/SI/P254

(C) 2009 Berger et al; licensee BioMed Central Ltd.

Assemblies of synchronously active neurons were suggested as the key mechanism for cortical information processing. Testing this hypothesis requires to observe large sets of neurons simultaneously, which is possible now due to recent advancements in electrophysiology. However, tools for analyzing such massively parallel data are lagging behind. Mere pairwise analysis is not sufficient to reliably detect synchronous spike patterns involving larger groups of neurons, and thus do not allow to conclusively identify assemblies. Instead methods that consider higher-order correlations are needed. Available tools for correlation analysis are not applicable, either because of the expected combinatorial explosion due to the required consideration of all individual spike patterns $[1,2]$, or, the methods are not designed to identify the specific set of assembly neurons [3-6].

Here we present a new method which detects and identifies neurons participating in assemblies, by combining the accretion method [7] with data mining approaches, in particular frequent item set mining (FIM). Thereby spike synchrony among groups of neurons is detected by the accretion approach: pairs of spike trains are tested for significant correlation and then reduced to new point processes containing only synchronized spikes. These processes are in turn correlated with single neuron spike trains and so on, until the maximal order of correlation is found. FIM algorithms help to fast and efficiently search the space of all neuronal subsets. However, FIM algorithms typically rely on a minimum support criterion to prune the search, since it guarantees soundness. In our framework, this criterion is not useful, since existence of higher correlation does not necessarily imply a frequent occurrence of spike patterns. We rather aim at selecting spike patterns that occur significantly more often than predicted by the spike and (lower order) coincidence rates. Therefore we employ the $\chi^{2}$ measure [7] in combination with the FIM algorithm still enabling to processes large data sets very efficiently. Using simulations of massively parallel spike processes that may contain various constellations of correlated activity (e.g., as A1, A3, A2 in figure 1; top panel) we illustrate that our algorithm is well suited for detecting and identifying groups of neurons exhibiting higher correlations. In particular, the approach is able to detect and separate neuronal groups of overlapping assemblies, irrespective of the number of assemblies individual neurons are participating in. The extracted assemblies are visualized by graphs where the nodes represent neurons and edges significant pairwise correlation (Figure 1, bottom panel). Thus we provide a powerful tool for the detection and identification of correlated groups of neurons. 


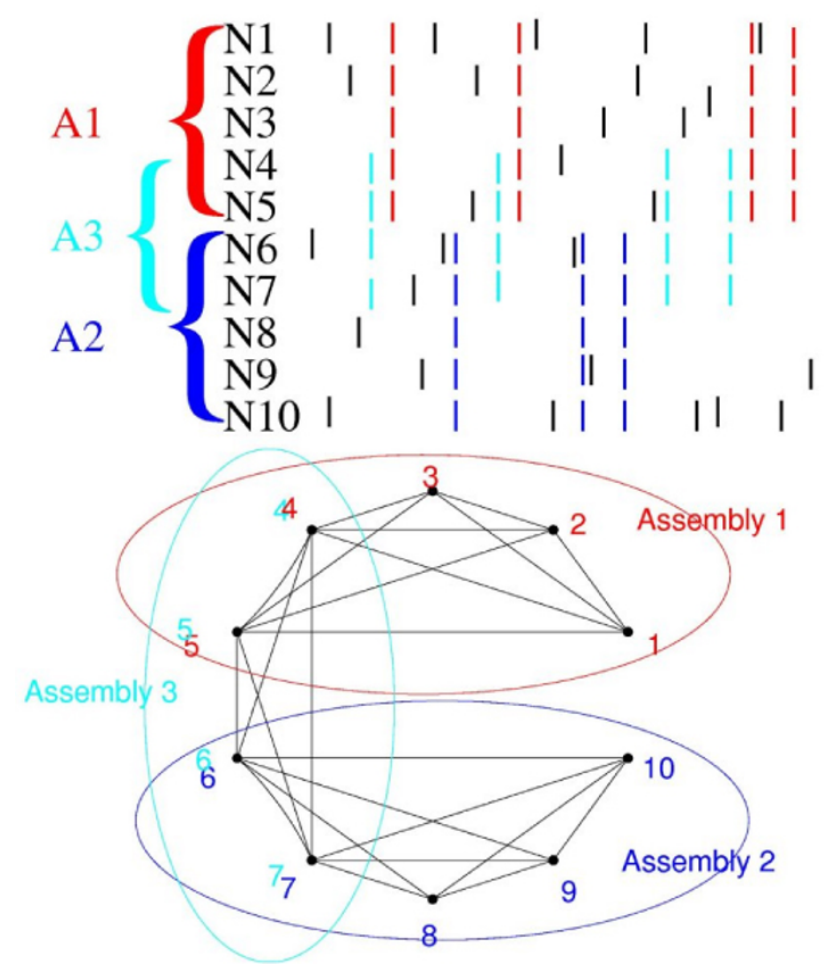

Figure I

\section{Acknowledgements}

Partially funded by BCCN Berlin (0IGQ04I3) and Helmholtz Alliance on Systems Biology.

\section{References}

I. Grün S, Diesmann M, Aertsen A: Neural Computation 2002, 14:43-80.

2. Shimazaki H, Amari S, Brown E, Grün S: Proc IEEE Intern Conf Acoustics, Speech, Signal Proc. (ICASSP) 2009.

3. Staude B, Rotter S, Grün S: Soc Neurosci, Program No. 103.9. San Diego 2007.

4. Grün S, Abeles M, Diesmann M: Lecture Notes in Computer Science 2008, 5286:96-II4.

5. Louis S, Grün S: CNS Meeting 2009.

6. Grün S, Berger D, Borgelt C: Proc IEEE Intern Conf Acoustics, Speech, Signal Proc. (ICASSP) 2009.

7. Gerstein GL, Perkel DH, Subramanian KN: Brain Research 1978, 140:43-62.
http://www.biomedcentral.com/1471-2202/10/S1/P254 